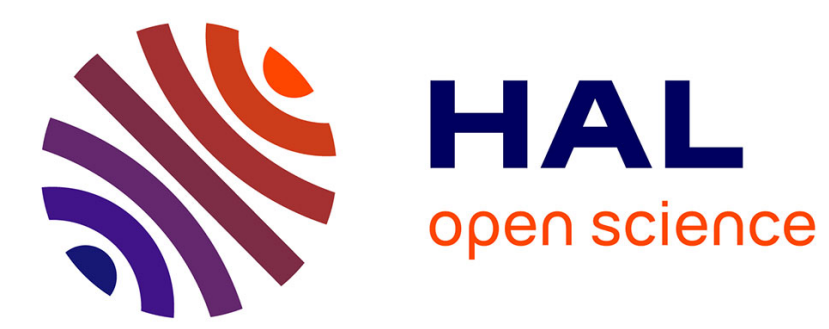

\title{
Ethnicisations ordinaires, voix minoritaires
}

Nicolas Jounin, Élise Palomares, Aude Rabaud

\section{To cite this version:}

Nicolas Jounin, Élise Palomares, Aude Rabaud. Ethnicisations ordinaires, voix minoritaires. Sociétés contemporaines, 2008, 70 (2), 10.3917/soco.070.0007 . halshs-01292282

\section{HAL Id: halshs-01292282 \\ https://shs.hal.science/halshs-01292282}

Submitted on 24 Mar 2016

HAL is a multi-disciplinary open access archive for the deposit and dissemination of scientific research documents, whether they are published or not. The documents may come from teaching and research institutions in France or abroad, or from public or private research centers.
L'archive ouverte pluridisciplinaire HAL, est destinée au dépôt et à la diffusion de documents scientifiques de niveau recherche, publiés ou non, émanant des établissements d'enseignement et de recherche français ou étrangers, des laboratoires publics ou privés. 


\section{CA I R N}

chercher : repérer : avancer

Cet article est disponible en ligne à l'adresse :

http://www.cairn.info/article.php?ID_REVUE=SOCO\&ID_NUMPUBLIE=SOCO_070\&ID_ARTICLE=SOCO_070_0007

Ethnicisations ordinaires, voix minoritaires

par Nicolas J OUNIN, Élise PALOMARES et Aude RABAUD

| Presses de Sciences Po | Sociétés contemporaines

$2008 / 02-n^{\circ} 70$

ISSN 1150-1944 | ISBN 9782724631296 | pages 7 à 23

Pour citer cet article :

- J ounin N., Palomares $n$ et Rabaud A., Ethnicisations ordinaires, voix minoritaires, Sociétés contemporaines 2008/02, $\mathrm{n}^{\circ} 70$, p. $7-23$.

Distribution électronique Cairn pour les Presses de Sciences Po.

(c) Presses de Sciences Po. Tous droits réservés pour tous pays.

La reproduction ou représentation de cet article, notamment par photocopie, n'est autorisée que dans les limites des conditions générales d'utilisation du site ou, le cas échéant, des conditions générales de la licence souscrite par votre établissement. Toute autre reproduction ou représentation, en tout ou partie, sous quelque forme et de quelque manière que ce soit, est interdite sauf accord préalable et écrit de l'éditeur, en dehors des cas prévus par la législation en vigueur en France. Il est précisé que son stockage dans une base de données est également interdit. 


\section{Nicolas Jounin / Élise Palomares / Aude Rabaud}

\section{ETHNICISATIONS ORDINAIRES,}

VOIX MINORITAIRES

I n'y a pas de minorités ici »:
ce fait social demeure
impensé dans le débat public comme dans la recherche française (Amiraux et Simon, 2006). Abdelmalek Sayad l'avait pourtant souligné dès 1979: toute migration de travail constitue, ipso facto, une migration de peuplement ; l'établissement familial des immigrés est cependant longtemps resté inconcevable, tant du point de vue des migrants que de celui de l'État et des sociétés de départ comme d'installation. Aujourd'hui, les descendants d'immigrés apparaissent collectivement distingués des autres Français en se voyant imputer - et en investissant parfois - des catégories ethniques diverses: selon «l'origine» géographique et/ou nationale, la culture, la religion, etc. Les mobilisations politiques, telles que la Marche pour l'Égalité et contre le Racisme (dite Marche des Beurs), posant la question du statut des immigrés et de la place de leurs descendants au sein de la société française, remontent à 1983. Elles se poursuivent jusqu'au récent «Appel des Indigènes de la
République » en janvier 2005, qui fait référence à un passé colonial français censément révolu. Durant les révoltes de l'automne 2005, outre les explications ouvertement racistes, la dénonciation de l'oppression raciale comme registre explicatif et/ou mobilisateur s'est largement déployée dans l'espace public.

En somme, dans le prolongement de la politisation intense des thèmes de la «maîtrise des flux » et de "l'intégration » dès le tournant des années quatrevingts que relevait Patrick Simon dans la présente revue en 1999, limmigration est plus que jamais au centre du débat public français. Immigration : ce terme est-il encore adéquat pour rendre compte des faits, des lieux et des gens dont on parle? En France, ce vocable recouvre trois ordres de faits: les migrations internationales, les relations interethniques et la constitution de minorités (Rea, Tripier, 2003). Cet usage dominant constitue l'un des paramètres du «cadre normatif qui organise les représentations nationales de l'altérité » (Streiff-Fenart, 1997; Lorcerie, 
1994), cadre idéologique statonational qui explique en partie la difficulté à penser le fait minoritaire.

Effectuant un pas de côté visà-vis des aspects les plus spectaculaires et médiatisés de la question, l'originalité de ce dossier réside dans le fait qu'il aborde de multiples formes d'ethnicisation ordinaire. Autrement dit, les contributions s'attachent à décrire et analyser la prégnance banale, normale, routinière des classements sociaux fondés sur l'origine. Quelles logiques sociales, économiques et spatiales ont contribué à les façonner? Comment s'articulent-t-elles avec le racisme ordinaire (Essed, 1991) ? Il sera peu question ici de criminalisation de l'immigration, de brutalités policières, d'invectives publiques ou de crimes à caractère raciste, mais de logiques sociales, économiques et institutionnelles, parfois animées des meilleures intentions, qui informent aussi l'expérience quotidienne des groupes minoritaires. De cette complexité des relations qui se tissent au jour le jour entre groupes majoritaires et minoritaires, il s'agit moins de dégager une analyse univoque des ferments de la colère que de démêler l'écheveau de relations asymétriques qui n'excluent ni la collaboration sur les lieux de travail, de loisir et de résidence, ni la confiance mutuelle, ni la convivialité, ni même l'amitié.
Pour les chercheurs, il est aujourd'hui moins question de dessiller les yeux des observateurs du monde social que de poursuivre une analyse rigoureuse des relations interethniques et du racisme, dans un domaine où de nombreuses friches empiriques et théoriques demeurent. En particulier, un point reste moins exploré: que nous apprend l'expérience individuelle et collective des acteurs sociaux en situation minoritaire?

Si les « voix minoritaires» ne sont jamais totalement absentes des travaux traitant des migrations internationales, des relations interethniques et du racisme, les spécialistes de ce champ s'accordent pour constater une carence relative, à la fois théorique et empirique, dans les recherches françaises.

Or, Danielle Juteau (1981) le souligne, les apports du discours minoritaire ont montré l'inadéquation (voire l'ineptie) de certains concepts et l'insuffisance de certains outils. C'est ainsi que des travaux pionniers ont rejeté successivement ou simultanément : le naturalisme (Mathieu, 1990 [1970] et 1977; Guillaumin, 1977, 1978), le psychologisme (Allport, 1954; Frazier, 1968 ; Van Den Berghe, 1967), le culturalisme (Breton, 1979; Franck, 1969) et l'économicisme (Laurin-Frenette, 1978) qui s'appliquaient - et parfois s'appliquent encore - à l'endroit 
des femmes et des minorités ethnicisées. La visibilité et la reconnaissance croissantes des productions théoriques des minoritaires ont introduit de nouvelles perspectives qui ont diversifié les connaissances en sciences sociales. Pour Danielle Juteau, de par leurs points de vue particuliers sur le social, les acteurs sociaux en situation minoritaire ont développé un regard spécifique soulignant, d'une part, les limites de l'objectivité de la connaissance et revendiquant le questionnement partiel de la réalité sociale, mettant à jour, d'autre part, la partialité de l'ensemble des analyses sociologiques, toute connaissance étant «située». Cette posture relativiste n'implique pas de renoncer à l'objectivation, elle vise à insister sur la vigilance nécessaire quant à l'universalisme scientifique abstrait, qui est, entre autres, ethno (et andro) centrique. Parmi un nombre considérable d'exemples confondants $^{1}$, citons ces deux là : les recherches françaises sur l'immigration n'ont pas pris en compte l'émigration avant les travaux d'Abdelmalek Sayad, tandis que le caractère sexué de la qualification n'apparaît qu'avec les recherches de Madeleine Guilbert en 1966.
Pourquoi parler de «minoritaires » ? Pourquoi pas dominés, exploités, opprimés? Parce que ces notions renvoient davantage à des configurations matérielles ${ }^{2}$, tandis que l'idée de minorité (au sens sociologique et non arithmétique) renvoie à un rapport qui « a deux faces, l'une concrète et l'autre idéologico-discursive», et « unit à l'intérieur du même univers symbolique minoritaires et majoritaires » (Juteau, 1999: 135). Parce que le groupe minoritaire ne voit pas seulement sa liberté restreinte en fait, n'est pas seulement l'objet d'un «traitement différent et inégal », selon la définition de Wirth (1945) de la minorité, mais qu'il est aussi limité en droit, à travers un discours et parfois un codage directement juridique qui lui impute une moindre capacité et le confine dans un particularisme, l'éloignant de l'universalité et de la généralité à laquelle prétend le majoritaire (Guillaumin, 2002 [1972] et 1985; Simon, 1983 ; Bertheleu, 2005). Les voix des minoritaires interviennent dans un ordre symbolique qui les spécifie, les isole, les amoindrit. Y prêter attention, c'est donc aussi interroger cet ordre. Et c'est peut-être entrevoir par où s'insinuent les résistances, justement, à la domination, l'exploitation, l'oppression.

1/ C'est l'adjectif qu'emploient Jacqueline Laufer, Catherine Marry et Margaret Maruani dans leur introduction de l'ouvrage collectif Le genre au travail paru en 2003. "En oubliant les femmes, ce n'est pas seulement de l'information que l'on perd, c'est de la connaissance que l'on déforme » écrivent-elle en citant quelques unes de ces «surprenantes omissions » et de leurs effets déformants.

2/ Le concept de « domination» fait partiellement exception, au moins depuis que Pierre Bourdieu lui a conféré une forte dimension symbolique, en radicalisant la perspective de Weber. 
Parler d'« ethnicisation », donc d'ethnicité, représente encore un certain défi dans le cadre des sciences sociales françaises. Ce n'est pourtant pas que l'« ethnie » en soit absente ; mais, précisément, après avoir été forgée dans le cadre d'une pensée racialiste à la fin du XIXe siècle ${ }^{3}$, elle était plutôt réservée aux populations anciennement colonisées justiciables d'une « ethnologie » (Amselle et M'Bokolo, 1985). Au regard de ce constat, il apparaît paradoxal que la réflexion sur la situation postcoloniale de la France suscite des réticences quant au risque d'introduire de manière incontrôlée des concepts étatsuniens qui seraient valables uniquement dans la formation sociale qui leur a donné naissance.

De plus, la production française (et plus encore francophone, en incluant les recherches canadiennes et belges) est désormais riche d'une clarification des concepts trop mal connue (Poutignat et Streiff-Fénart, 1995; Martiniello, 1995 ; Juteau, 1999 ; De Rudder, Poiret et Vourc'h, 2000 ; Rea et Triper 2003; Bastenier 2004; Simon, 2006). Elle emprunte à Max Weber l'accent mis sur les processus ainsi que le sens donné par les acteurs à leurs pratiques, au marxisme un matérialisme qui ne se réduit pas aux seules questions économiques, et à la sociologie américaine une approche interactionniste des phénomènes sociaux.

Le recours au concept d'ethnicisation est un moyen, pour les chercheurs, de spécifier une posture théorique et épistémologique à la fois dynamique, constructiviste et relationnelle. Dans un nombre croissant d'usages sociaux, l'ethnicisation constituerait une dérive pathologique des relations sociales (Bertheleu, 2007). Ce concept ne vise pourtant pas à dénoncer une évolution nuisible aux «valeurs républicaines », ainsi qu'il a pu être conçu au travers du prisme d'un «nationalisme méthodologique $^{4} »$. Il ne constitue pas non plus un euphémisme savant pour désigner le racisme. Il est question d'ethnicisation des rapports sociaux lorsque des catégories ethniques constituent des « référents déterminants de l'action et dans l'interaction, par opposition aux situations dans lesquelles ces imputations ne constituent qu'un référent parmi d'autres du rôle, du statut, et en dernière instance, de la position hiérarchique dans

3/ D’après P.J. Simon (1993) le terme a été « utilisé pour la première fois en France par Vacher de Lapouge (Les sélections sociales, 1896), repris par Alfred Fouillée (Psychologie du peuple français, 1914), son sens précisé en 1920 par Regault, différenciant l'ethnie (critère linguistique) de la race (critère anatomique), et son usage popularisé par Georges Montandon ». Ce dernier publie en 1935 son ouvrage, L'ethnie Française, qui, pour S. Jarnot, pose les « jalons d'une "ethno-raciologie" », prélude à son engagement auprès des nazis dès 1938, qui lui confieront en 1943 la direction de l'Institut d'Études des Questions Juives et Ethnoraciales (Jarnot, 2000).

4/ C'est l'expression qu'emploie Smith (1981) dans le cas britannique, et que reprend Bastenier (2004) pour caractériser les travaux français centrés sur une conception normative de l'« intégration ». 
les classements sociaux» (De Rudder, Poiret et Vourc'h, 2000). La pertinence sociale des catégories ethniques est alors centrale ; elles deviennent l'axe principal à partir duquel sont définies et interprétées les situations.

Cet outil permet d'abandonner toute définition substantialiste de l'ethnicité selon laquelle les groupes ethniques existent par eux-mêmes puis, éventuellement, établissent des contacts, au profit d'une perspective relationnelle: un groupe ne devient ethnique que lorsque ses membres viennent à se distinguer en revendiquant pour eux, ou en assignant à d'autres individus, une commune origine (Poutignat et Streiff-Fénart, 1995). Au même titre que la race et le sexe, les catégories ethniques ont longtemps été envisagées comme se fondant sur des différences objectives, mesurables. C'est Max Weber (1995 [1922]), le premier, qui va s'écarter radicalement de la vision essentialiste de l'appartenance ethnique. Selon lui, les groupes ethniques se caractérisent moins par des traits objectifs (territoire, religion, langue, rite...) que par une croyance partagée en des origines communes.

C'est plus particulièrement dans son analyse de la communalisation que Weber montre en quoi le fait ethnique est nécessairement relationnel. Il ouvre ainsi la voie à cette approche des groupes et des frontières ethniques comme se construisant dans la relation. On doit ensuite à Everett Hughes (1996 [1948]) le développement de cette perspective. En étudiant les relations interethniques et les différences de classe entre les anglophones et les francophones du Canada, il montre comment les groupes ethniques sont le produit de processus d'identification.

Cette approche sera poursuivie par l'anthropologue Frederik Barth qui livre un texte de rupture en 1969 en proposant d'étudier l'organisation sociale et politique de la différence culturelle. Son approche interactionniste l'amène à montrer que c'est la construction, la disparition et le maintien de frontières ethniques qui produisent de la différenciation culturelle. Pour Frederik Barth (1995 [1969]), bien plus que les caractéristiques culturelles ou physiques, réelles ou supposées, censées être propres à un groupe ethnique, c'est la dichotomie entre «Eux» et « Nous » qui joue un rôle central dans la constitution et la définition des contours de ce groupe. Ce sont ces rapports réciproques - bien qu'asymétriques - entre une altérité et une identité collectives qui intéressent la sociologie des relations interethniques.

Nous considérons que le concept d'ethnicisation est un outil offrant la possibilité d'interpréter l'omniprésence de catégories de 
classement ethniques produites dans et par des rapports sociaux inégalitaires. La vigilance antinaturaliste est a fortiori nécessaire pour s'intéresser au processus plus spécifique de fabrique de l'altérité qu'est la racisation. En tant que mode radicalisé de l'ethnicisation, la racisation absolutise la différenciation selon l'origine, la culture et l'érige en catégorie immuable et définitive ; elle implique un principe explicatif ultime : elle naturalise la différence (Guillaumin, 1977 ; De Rudder, 1991 ; Fassin et Fassin, 2006).

Dans cette perspective, il est question de travailler sur des processus et non sur des états, sur la production de l'altérité et de différences, sur les catégorisations ethniques et « raciales » dans des cadres institutionnels ou routiniers, sur leurs effets dans l'organisation des relations sociales et l'orientation de l'action.

Si les mises au point conceptuelles sont toujours nécessaires, l'intérêt d'étudier les relations interethniques, qui faisaient déjà l'objet d'un dossier dans la présente revue en 1990, n'est plus à démontrer. Depuis les années quatre-vingts, de nombreux travaux ont analysé la gestion de la coexistence sur un même territoire de populations aux appartenances sociales et ethniques différenciées ${ }^{5}$. Une part importante de cette production française dans le champ de la sociologie urbaine s'est centrée sur les pratiques d'occupation et d'appropriation de l'espace public par les populations installées dans des espaces résidentiels aux dynamiques de peuplement spécifiques (centres villes anciens, grands ensembles). L'attention est portée sur les enjeux matériels, symboliques et politiques de la coexistence entre « autochtones et immigrés » (De Rudder, 1987 ; Rinaudo, 2000 ; Rabaud, 2003). S'inspirant pour une part des apports de l'École de Chicago, ces travaux insistent sur les modalités d'accès à certaines ressources matérielles, à commencer par le logement (De Rudder, 1995; Simon, 1999 ; Tanter et Toubon, 1999). Les chercheurs analysent des formes de cohabitation inédites qu'engendrent les opérations de rénovations et de requalification urbaines (Simon, 1995 ; Grafmeyer, 1999), la territorialisation et les regroupements communautaires (Benveniste, 2002) ou la spécialisation professionnelle et les phénomènes d'ethnic business (Ma Mung 1992 ; Raulin, 2000).

À partir des années quatrevingt-dix, un nombre croissant de travaux français a questionné les processus d'ethnicisation et

5/ Pour une analyse détaillée et très complète du développement des recherches françaises sur « l'urbain » et «l'ethnique », se reporter à De Rudder (1990). Dans cet article, l'auteur étudie l'évolution thématique et méthodologique des travaux français depuis les années soixante-dix et offre une importante bibliographie des travaux produits jusqu'à la fin des années quatre-vingts. 
de racisation à l'œuvre dans et par les institutions. Les études portant sur les discriminations dans l'emploi, la santé ou, dans une moindre mesure, l'éducation et le logement se multiplient et se diversifient (Dhume et Sagnard-Haddaoui, 2006 ; Carde, Fassin, Ferre et Musso-Dimitrijevic, 2001 ; Simon, 2001 ; VEI Enjeux, 2002, Tissot, 2005). Les enquêtes prenant pour objet d'étude le rôle des institutions publiques dans la production de différences ethniques (Poiret, 1996; Geisser, 1997; Wieviorka, 1998 ; de Barros, 2005 ; Palomares, 2005) doivent beaucoup aux travaux de chercheurs étrangers (Ireland, 1994; Hargreaves, 1995). En 1998, Marco Martiniello codirige avec MarieAntoinette Hily un dossier de la Revue Européenne des Migrations Internationales qui interroge la place des minorités dans les institutions politiques européennes ${ }^{6}$, tandis que paraît Immigration et racisme en Europe, sous la direction d'Andrea Rea. Dans le champ scolaire, depuis 1996, des travaux sur l'ethnicisation des rapports sociaux sont produits selon un rythme très supérieur à celui de la décennie précédente (Rinaudo, 1998; Lorcerie, 2003; Payet, 2002, 2007). Il en va de même pour les domaines de la santé (Fassin, 2001 ; Cognet, 2004 ; Tersigni, 2008) et du travail (Bataille,
1997; De Rudder, Poiret et Vourc'h, 2000 ; Jounin, 2008).

L'important, à présent, est de continuer à avancer dans cette voie, en linscrivant dans le plan général de la sociologie et non en la circonscrivant comme un isolat de recherches exotiques. Pour ce faire, il est nécessaire de réfléchir à l'articulation des rapports interethniques aux autres rapports sociaux, et d'inscrire ces rapports dans les termes généraux de la domination et de la résistance à la domination.

La nécessité de croiser, d'articuler, de combiner, d'imbriquer, d'« intersectionner » les rapports sociaux est aujourd'hui largement partagée (Juteau, 1999; Poiret, 2005; Falquet, Lada et Rabaud, 2006; Dorlin, 2008; Kergoat, 2008; Scrinzi, 2008.) Dans le sillage des contributions pionnières de Colette Guillaumin sur le sexisme et le racisme et de Danièle Kergoat sur l'articulation du sexe et de la classe sociale en 1978, cette démarche a nourri une littérature abondante et discutée. Nous ne chercherons pas ici à prendre position dans ces débats, sinon pour réaffirmer l'importance des enquêtes empiriques et pour récuser la primauté a priori d'un rapport social (le sexisme, le racisme, ou le rapport de classe) sur les autres. La question qui

6/ Sur ce point, mentionnons également le livre d'O. Masclet, La Gauche et les cités : enquête sur un rendezvous manqué paru en 2003 chez La Dispute : enquête ethnographique à l'appui, l'auteur montre « comment une municipalité n'a pas pu ou n'a pas su reconnaître politiquement les enfants d'immigrés ». 
relie les spécialistes de chacun de ces trois grands rapports sociaux est celle de l'articulation concrète entre des signifiés (les rapports de pouvoir et de production) et des signifiants (schèmes d'interprétation en termes de race, sexe, classe...).

Rapport entre administrés et responsables politiques; rapport entre services sociaux et leurs usagers; rapport entre ouvriers et chefs: tous ces rapports, décrits au fil des articles, ont leur propre logique, en partie irréductible aux rapports interethniques. Mais ils se croisent et se redéfinissent avec ces derniers. Si l'ethnicisation des rapports sociaux est une réalité massive, l'erreur serait cependant de la tenir pour acquise pour chaque espace considéré, sans s'interroger sur la manière dont elle s'actualise ou se transforme. Les frontières des catégories ethniques et le contenu donné aux catégories connaissent des altérations selon les contextes.

Il en va de même pour l'idéologie raciste, à la fois contraignante et relativement souple, redéfinie par les acteurs selon leur point de vue, leurs intérêts: un maire, une assistante sociale ou une commerciale d'intérim du bâtiment peuvent tous trois tenir des discours racistes, c'est-à-dire des discours d'infériorisation essentialistes fondés sur l'origine, mais d'une teneur pourtant différente. Car c'est le rapport de leurs cibles tantôt à la politique, tantôt à l'aide sociale, tantôt au travail qui est visé, et les stéréotypes mobilisés peuvent alors être incohérents entre eux. La logique du racisme n'est pas à rechercher seulement en interne, dans la doctrine même, mais en situation, dans son lien avec les contingences que le locuteur tente de mettre en ordre en recourant à la transcendance de natures supposées. En France, le racisme a longtemps été exclusivement analysé en tant qu'idéologie, appelant une condamnation morale sans appel. Prolongeant les apports théoriques denses et anciens sur cette question, l'enjeu de ce dossier consiste à explorer les manifestations quotidiennes de racisme ordinaire, indépendamment de l'adhésion à (ou du rejet de) la doctrine raciste par des acteurs sociaux.

Enfin, le point de vue commun aux articles qui constituent ce dossier déborde largement le champ des relations interethniques: nous considérons que « les relations de domination sont, en même temps, des relations de résistance » (Scott, 1990 : 45). Cette idée peut sembler triviale, mais elle rompt avec certaines perspectives qui voient la domination s'inscrire dans l'esprit et dans le corps des dominés comme sur une page vierge. Elle permet aussi, à la suite de Foucault notamment, de ne pas limiter la conception de 
ce qu'est la résistance aux seules confrontations organisées, porteuses d'une alternative globale. La résistance est tout autant contournement, dérobade, fuite, qu'affrontement. Sa possibilité est inscrite dans toute relation de pouvoir et, en attendant son actualisation, la mise en conformité peut n'être qu'un accommodement temporaire, et non une résignation, encore moins une croyance en la légitimité de la situation.

Cette résistance éclatée, ou différée, souvent inaperçue quoique quotidienne, est annoncée par des discours que les minoritaires tiennent dans certains espaces protégés, dans les coulisses des scènes d'interaction avec ceux qui les «minorisent $»$ (coulisses qui prennent la forme, dans les articles qui suivent, de vestiaires de chantier, de préparatifs d'une réunion publique organisée par la mairie, du chemin qui mène au bureau de l'assistante sociale...). S'intéresser à ces discours clandestins, ces «hidden transcripts" ainsi que les nomme James C. Scott (1990), c'est vouloir restituer les " effets hérétiques de la parole », les « effets d'écart du social à luimême » (Cingolani, 2003 : 116). C'est rechercher les manifestations d'une réflexivité qui permet aux acteurs de préserver un décalage entre ce qu'ils font et ce qu'ils sont, et qui ce faisant guide le sociologue dans son travail d'objectivation.
Dans les contributions réunies ici, les auteurs s'attachent à montrer que l'examen des multiples formes de résistances à l'oppression véhiculée par les discours et les pratiques racistes et sexistes dans la vie quotidienne contribue à produire des connaissances nouvelles et à enrichir la réflexion existante sur les processus de domination. Ce questionnement se décline à travers des contributions portant sur les relations sociales ordinaires de travail, de voisinage, aux guichets de l'action sociale et dans les espaces publics urbains. Les articles proposés sont issus d'enquêtes de terrain, fondées sur l'insertion personnelle du chercheur au sein des mondes étudiés.

Nicolas Jounin analyse les relations de domination et de résistance entre les immigrés maghrébins et ouest-africains relégués dans les positions les moins valorisées du bâtiment et leurs employeurs. D'une part, la législation sur le séjour les rend vulnérables et le discours raciste de leurs employeurs affirme leur illégitimité et leur inutilité pour mieux légitimer leur exploitation économique; au quotidien sur les chantiers d'autre part, l'oppression prend les formes d'humiliations, de mensonges, d'agressivité verbale, de surnoms et d'insultes. "De toute façon si tu meurs, il y a plein de Noirs" dit en substance un chef d'équipe à un manœuvre. Ces menaces 
réitérées ont pour fonction de rappeler le statut précaire (du séjour comme de l'emploi) de ceux qui en sont les cibles, statut qui rend la contestation organisée de l'exploitation improbable. Dans ce contexte, la résistance n'a que peu de prises sur les instances qui contrôlent réellement la situation; pour ces travailleurs, les maillons les plus visibles sont les intermédiaires, commercial d'intérim et chef d'équipe. La franche insoumission à l'égard de ces derniers se paye cher: elle se solde par le renvoi immédiat qu'autorise l'absence de contrat à durée indéterminée. Les manœuvres participent donc au flot de plaisanteries et de «vannes » sexistes et racistes mais leur position suppose d' "encaisser sans rechigner », même quand elles vont trop loin à leurs yeux. La résistance passe par un discours souterrain de la révolte justifiant les pratiques de ralentissement, le travail bâclé, les vols sur le chantier, exprimé notamment par la sentence: "c'est pas un métier", niant au passage leur propre savoir faire. L'absentéisme constitue l'un des modes privilégiés du répertoire de cette résistance silencieuse, dût-il passer par des accidents provoqués par l'ouvrier lui-même.

La situation minoritaire de femmes originaires du Mali analysée par Elise Palomares s'inscrit dans une relation ancienne avec une municipalité de gauche. Sur ces lieux de résidence, la donne apparaît d'emblée différente. Les idéaux de solidarité et d'antiracisme occupent une place centrale dans la construction d'une nouvelle identité locale qui ne s'adosse plus à la référence à la classe ouvrière. Les politiques locales de cette ville de l'ancienne Ceinture Rouge s'établissent désormais en référence constante au mythe républicain national («France terre d'accueil »). Interprétant de ce fait tout regroupement ethnique organisé comme les prémices d'un dangereux communautarisme, la municipalité s'appuie pourtant au quotidien sur un partenariat avec les associations de migrants Maliens. La minorisation prend ici la forme d'une bienveillante sollicitude collective : les Maliens ont été progressivement institutionnalisés comme un groupe posant un problème social spécifique du fait d'une supposée distance culturelle, nécessitant des actions volontaristes en vue de leur «intégration » ainsi que celle de leurs enfants. Dans ce contexte, la dynamique associative regroupant Françaises et Maliennes constitue un cadre contraignant et habilitant. Aussi les femmes originaires du Mali ne se risquent-elles pas à faire porter le débat sur les pratiques discriminatoires et les discours racistes qu'elles rencontrent au quotidien, bien que les ayant clairement identifiés comme tels. Dans les coulisses, elles s'interrogent sur l'injonction à l'intégration faite à leurs enfants: "On ne 
devrait pas parler d'intégration pour eux: qu'est-ce que cela veut dire?» Surtout, sur ce même mode mineur, elles mettent l'accent sur la racisation dont l'ensemble du groupe fait l'objet. Leur expérience de la «ligne de couleur» remet en cause l'idée qu'il s'agirait là d'une différenciation ethnique liée au contexte migratoire, fondée sur l'origine nationale, culturelle ou religieuse, variant souplement au fil des années et au gré des situations; elle repose la question du statut de leur descendance dans l'espace national.

De prime abord, la situation des migrantes marocaines âgées en France est à la croisée de dominations coextensives qui peuvent apparaître écrasantes. À contre-pied de cette évidence, l'article de Fatima Ait Ben Lmadani fait la lumière sur les multiples tactiques de résistance qu'elles mettent en œuvre dans leurs relations avec des professionnels de l'action sociale. L'auteure se focalise sur les réponses aux injonctions à la participation et à l'autonomie de la part de ces femmes, rendues invisibles dans la société française. Outre ces attentes normatives à leur égard, ces migrantes marocaines âgées doivent également gérer des conditions de logement précaires, des revenus très faibles, un réseau de sociabilité plus ou moins dense, un état de santé incertain. Ces facteurs structurent le système de contraintes auquel elles doivent faire face. Contournement, détournement ou affrontement, Fatima Ait Ben Lmadani montre la diversité des registres de résistance qu'elles mobilisent individuellement, au quotidien, en matière d'accès au logement, aux loisirs ou à l'autonomie financière. Détournement des objectifs de l'aide sociale, qu'elles considèrent comme un droit au regard d'une longue vie de travail non reconnue comme telle; mise en scène de soi par le recours à des stéréotypes culturalistes pour accéder aux ressources; refus d'aller en maison de retraite en arguant de l'injonction à l'autonomie à laquelle elles sont régulièrement confrontées : au-delà des apparences, en pratique et en acte, ces femmes résistent aux représentations et aux assignations qui les enferment. Le texte fait apparaître leurs tentatives pour transformer leurs expériences du mépris, de l'humiliation et du discrédit en ressources tant matérielles que symboliques pour bénéficier d'un surcroît de légitimité dans la société d'installation comme au sein de leur société d'origine.

En conclusion, dans le travail d'enquête comme d'interprétation, l'attention portée à ces discours et pratiques mineures ne revient pas à verser dans un populisme qui commanderait de quêter la parole « authentique » des minoritaires. D'une part, parce qu'il n'y a pas une parole 
unique: l'articulation entre les situations vécues et des mots pour les dire est multiple, en témoignent les incessantes discussions et altercations inter et intra minoritaires (Rabaud, 2003). D'autre part, parce que si ces discours produisent de la réflexivité, donc de l'extériorité, ils ne viennent pas de l'extérieur et ne peuvent être radicalement étrangers à l'idéologie majoritaire. Ils peuvent même subvertir l'ordre raciste tout en étant racistes dans leur énoncé, comme l'étaient la «négritude » de Senghor ou les mythes fondateurs de la Nation de l'Islam au temps de Malcolm X (Malcolm X, 1993).

Mais en citant ces illustres exemples ou d'autres « mobilisations ethniques» (Bourgois, 1993, Le Texier, 2007), on se réfère encore à des résistances déjà articulées dans une réflexion intellectuelle systématisée et/ou une action organisée. Les articles qui suivent analysent plutôt des voix minoritaires inorganisées, qui accompagnent des résistances relativement dispersées, au sein d'interactions de petite envergure. Il ne s'agit pourtant pas d'en rester au témoignage, ni de prétendre répercuter la parole de ceux qui ne l'ont pas, ou encore d'exhumer un résidu de pratiques et de discours tôt écrasés par les structures sociales. Nous pensons plutôt que, dans cette accumulation de situations et de contournements, qui certes font peu de bruit et ne laissent pas de traces directes, il est possible de lire les potentialités du présent et de comprendre un peu mieux le changement social.

\section{RÉFÉRENCES BIBLIOGRAPHIQUES}

ALLPORT G., 1954

AMIRAUX V., SIMON P., 2006

AMSELLE J.-L., M'BOKOLO E., 1985

BARTH F., 1995 [1969]

BASTENIER A., 2004

BATAILLE P., 1997
The nature of prejudice, Cambridge : Mass, Addison-Wesley.

There are no minorities here: cultures of scholarship and public debate on immigrants and integration in France, International Journal of Comparative Sociology, vol. 47 (3-4), p. 191-215.

Au cœur de l'ethnie, Paris : La Découverte.

Les groupes ethniques et leurs frontières, In : Poutignat P., Streiff-Fenart J., Théories de l'ethnicité, Paris : PUF, p. 207-270.

Qu'est-ce qu'une société ethnique? Ethnicité et racisme dans les sociétés européenne d’immigration, Paris : PUF.

Le racisme au travail, Paris : La Découverte. 
BARROS (DE) F., 2005

BENVENISTE A., 2002

BERTHELEU H., 2005

BERTHELEU H., 2007

BOURGOIS P., 1993

BRETON R., 1979
Des « Français musulmans d'Algérie » aux « immigrés ». L'importation de classifications coloniales dans les politiques de logement en France (1960-1970), Actes de la Recherches en Sciences sociales, $n^{\circ}$ 159, septembre, p. 26-45.

Figures politiques de l’identité juive à Sarcelles, Paris : L'Harmattan.

Les rapports sociaux constitutifs des groupes minoritaires et majoritaires : une approche sociologique. In : Martinez F., Lichaud, M.-C. (dir.), Minorité(s). Construction idéologique ou réalité ?, Paris : PUR, p. 19-26.

Sens et usages de l'« ethnicisation ». Le regard majoritaire sur les rapports sociaux ethniques, Revue Européenne des Migrations Internationales, vol. 23, n ${ }^{\circ}$ 2, p. 7-28.

La mobilisation ethnique, Actes de la Recherche en Sciences Sociales, vol. 99, n 9, p. 53-64.

Ethnic stratification Viewed from Theoretical Perspectives In : Curtis J.E. and Scott W.E. (ed.), Social Stratification in Canada, Scarborough : Ont. Prentice-Hall.

CARDE E., FASSIN D., FERRE N., Un traitement inégal : les discriminations dans l'accès aux MUSSO-DIMITRIJEVIC S. (dirs.), soins, Bobigny : Centre de Recherche sur les Enjeux 2001 contemporains en santé publique (CRESP).

CINGOLANI P., 2003

La République, les sociologues et la question politique, Paris : La Dispute.

L'accès à l'emploi dans les services de santé : l'effet de la dimension ethnique. Étude du groupe des auxiliaires familiaux et préposés aux bénéficiaires à Montréal, In : Renaud J., Germain A., Leloup X. (eds.), Racisme et discrimination : permanence et résurgence d'un phénomène inavouable, Lyon : PUL, p. 60-86.

DE RUDDER V. (en coll. avec GUILLON M.), 1987

DE RUDDER V., 1991

DE RUDDER V., 1995

Autochtones et immigrés en quartier populaire : du marché d'Aligre à l'îlot Châlon, Paris : L'Harmattan.

Le racisme dans les relations interethniques, L'Homme et la Société, vol. 102, p. 75-90.

La ségrégation est-elle une discrimination dans l'espace? Éléments de réflexion sur les relations interethniques, In : Gallissot R., Moulin B. (dir.), Les quartiers de la ségrégation. Tiers monde ou quart-monde? Paris : Karthala/Institut Maghreb-Europe, p. 11-30.

DE RUDDER V., 1990

Notes à propos de l'évolution des recherches françaises sur « l'étranger dans la ville», In : Simon-Barouh I., Simon, P.-J. (dirs.), Les étrangers dans la ville, Paris : L'Harmattan, p. $60-80$.

DE RUDDER V., POIRET C., VOURC'H F., 2000

L’inégalité raciste. L’universalité républicaine à l'épreuve, Paris : PUF.

DORLIN E. (dir.), (à paraître 2008) Sexe, classe, race. Pour une épistémologie de la domination, Paris : PUF.

DHUME F., Les Discriminations raciales à l'emploi. Une synthèse SAGNARD-HADDAOUI N., 2006 problématique des travaux, Montpellier : ISCRA.

ESSED P., 1991
Everyday racism : an interdisciplinary theory, London : Sage publications. 
FALQUET J., LADA E., RABAUD A. (Ré)articulation des rapports sociaux de sexe, classe et (dir.), 2006 « race ». Repères historiques et contemporains, Cahiers du CEDREF

FASSIN D., 2001

Une double peine. La condition sociale des immigrés malades du sida, L'Homme. Revue française d'anthropologie, 160, p. 137-162.

FASSIN D., FASSIN E. (dirs.), 2006 De la question sociale à la question raciale ? Représenter la société française, Paris : La Découverte.

FOURIER M., VERMES G. (dirs.) Ethnicisation des rapports sociaux. Racismes, nationalismes, ethnicismes et culturalismes, Paris : L'Harmattan.

FRANCKA.G., 1969

Sociology of Development and Under Development of Sociology, In : Latin America : Underdevelopment or Revolution, New York : Moderne Reader, p. ???

FRAZIER F., 1968

Theorical Structure of sociology and Sociological Research, In : Edwards G.F. (ed.) E. Franklin Frazier : on race relations, Chicago : University of Chicago Press.

GEISSER V., 1997

Ethnicité républicaine : les élites d’origine maghrébine dans le système politique français, Paris : Presses de la FNSP.

GRAFMEYER Y., 1999

La coexistence en milieu urbain : échanges, conflits, transactions, Recherches sociologiques, Sociologie de la ville, vol. XXX, n 1, p. 157-176.

GUILBERT M., 1966

GUILLAUMIN C., 1977

GUILLAUMIN C., 1978

GUILLAUMIN C., 1985

Les fonctions des femmes dans l'industrie, La Haye, Mouton.

Race et nature : système des marques, idée de groupe naturel et rapports sociaux, Pluriel-Débat, 11, p. 39-55.

Pratique du pouvoir et idée de nature. (2) Le discours de la nature, Questions féministes, 3, p. 5-30.

Sur la minorité, L’homme et la société, vol. 77-78, p. 101-109.

GUILLAUMIN C., 2002 [1972]

L’idéologie raciste. Genèse et langage actuel, Paris : Gallimard.

HILY M.-A., MARTINIELLO. M. (dir.), 1998

Immigrés et minorités ethniques dans l'espace politique européen. Revue Européenne des Migrations Internationales, vol. $14, n^{\circ} 2$

HARGREAVES A.G., 1995

HUGHES E.C., 1996 [1948]

Immigration, "race" and ethnicity in Contemporary France, London : Routledge.

La rencontre des groupes ethniques et des cultures. L'étude des relations ethniques, In : Chapoulie J.-M. (dir.), Le regard sociologique : essais choisis. Paris : Éd. EHESS, p. 201-207.

IRELAND P., 1994

The Policy Challenge of Ethnic Diversity : Immigrant Politics in France and Switzerland, Cambrigde, Mass., Harvard University Press.

JARNOT S., 2000

Une relation récurrente : science et racisme : l'exemple de l'Ethnie française. Cahiers du CERIEM, n 5, p. 17-34.

JOUNIN N., 2008

Chantier interdit au public. Enquête parmi les travailleurs du bâtiment, Paris : La Découverte.

JUTEAU D., 1999

L'ethnicité et ses frontières, Montréal : Presses universitaires de Montréal.

JUTEAU-LEE D., 1981

Visions partielles, visions partiales : visions des minoritaires en sociologies, Sociologie et Sociétés, vol. 13, $\mathrm{n}^{\circ} 2$, p. 33-47. 
KERGOAT D., (à paraître 2008)

KERGOAT D., 1978

LAUFER J., MARRY C. MARUANI M. (dir.), 2003

LAURIN-FRENETTE N., 1978

LE TEXIER E., 2006

LORCERIE F., 1994

LORCERIE F., 2003

MA MUNG E., 1992

MARTINIELLO M., 1995

MASCLET O., 2003

MATHIEU N.-C., 1977

MATHIEU N.-C., 1990 [1970]

PALOMARES E., 2005

PAYET J.-P., 2002

PAYET J.-P., 2007

POIRET C., 1996

POIRET C., 2005

POUTIGNAT P., STREIFF-FENART J., 1995
Dynamique et consubstantialité des rapports sociaux, In : Dorlin E. (dir.), Sexe, classe, race. Pour une épistémologie de la domination, Paris: PUF.

Ouvriers = ouvrières ? Propositions pour une articulation théorique de deux variables : sexe et classe sociale, Critiques de l'économie politique, nouvelle série, n 5, p. 65-97.

Le genre au travail. Les sciences sociales à l'épreuve des différences de sexe, Paris, La Découverte/MAGE

Production de l'État et formes de la nation, Montréal : Nouvelle optique.

Quand les exclus font de la politique. Le barrio mexicain de San Diego, Californie, Paris : Presses de la FNSP.

Les sciences sociales au service de l'identité nationale, In : Martin D.-C. (dir.), Cartes d'identité. Comment on dit " nous » en politique?, Paris : Presses de la FNSP, p. 245.281 .

L'école et le défi ethnique : éducation et intégration, Issy-les-Moulineaux : ESF éditeur.

L'expansion du commerce ethnique : Asiatiques et Maghrébins dans la région parisienne. Revue Européenne des Migrations Internationales, vol. 8, nº 1, p. 39-59.

L'ethnicité dans les sciences sociales contemporaines, Paris : PUF.

La Gauche et les cités : enquête sur un rendez-vous manqué, Paris : La Dispute.

Paternité biologique, maternité sociale..., In : Michel A. (ed.), Femmes, sexisme et sociétés, Paris : PUF, p. 39-48.

Notes pour une définition sociologique des catégories de sexe, In : Mathieu N.-C., 1990, L'anatomie politique. Catégorisations et idéologie du sexe, Paris : Côté-femmes, p. 17-41.

L'ethnicisation des politiques locales, Contretemps, $\mathrm{n}^{\circ} 13$, p. 93-102.

L'ethnicité c'est les autres. Formes et enjeux de la relation de l'école aux milieux disqualifiés, VEI Enjeux, nº 6, p. 55-64.

L'ethnicisation des rapports scolaires et la discrimination ethnique au sein de l'école : un bilan des travaux en France et en Europe. Site GRS-CNRS

$<$ http://recherche.univ-lyon2.fr/grs/index.php?page=29 \& notice $=46>$

Familles africaines en France. Ethnicisation, ségrégation et communalisation, Paris : L'Harmattan/CIEMI.

Articuler les rapports de sexe, de classe et interethniques : quelques enseignements du débat nord-américain, Revue Européenne des Migrations Internationales, vol. 21, $\mathrm{n}^{\circ} \mathrm{l}$, p. 195-226.

Théories de l'ethnicité, Paris : PUF. 
RABAUD A., 2003

RAULIN A., 2000

REA A. (dir.), 1998

REA A., TRIPIER M., 2003

RINAUDO C., 1998

RINAUDO C., 2000

SAYAD A., 1979

SCOTT J.C., 1990

SCRINZI F., 2008

SIMON P., 1995

SIMON P. (dir.), 1999

SIMON P., 2001

SIMON P.-J., 1983

SIMON P.-J., 1993

SIMON P.-J., 2006

SIMON-BAROUH I. DE RUDDER V. (dirs.), 1999

SMITH A., 1981

Sociétés Contemporaines, 1990

STREIFF-FENART J., 1997

TANTER A., TOUBON J.-C
Le souci du voisinage. Organisation politique et associative de la vie locale et formes ordinaires de l'échange dans un grand ensemble, Thèse de doctorat de sociologie, Université Paris 7 Diderot.

L'ethnique est quotidien. Diasporas, marchés et cultures métropolitaines, Paris : L'Harmattan.

Immigration et racisme en Europe, Bruxelles : Éd. Complexe. Sociologie de l'immigration, Paris : La Découverte.

L'imputation de caractéristiques ethniques dans l'encadrement de la vie scolaire, Revue Européenne des Migrations Internationales, vol. 14, n ${ }^{\circ} 3$, p. 27-43.

L'ethnicité dans la cité, Paris : L'Harmattan.

Qu'est-ce qu'un immigré ?, Peuples Méditerranéens, vol. 7, p. 3-23.

Domination and the arts of resistance, New Haven and London : Yale university press.

Quelques notions pour penser l'articulation des rapports sociaux de " race », de classe et de sexe, In : Falquet J., Rabaud A., Freedman J., Scrinzi F., Femmes, genre, migrations et mondialisation : un état des problématiques, Cahiers du CEDREF, p. 81-99.

La société partagée. Relations interethniques et interclasses dans un quartier en rénovation, Belleville, Paris XX Cahiers internationaux de sociologie, vol. XCVIII, p. 161-190.

Immigration et politiques de l'habitat, Sociétés Contemporaines, $\mathrm{n}^{\circ} 33-34$.

Les discriminations raciales et ethniques dans l'accès au logement social, Paris : Groupe d'Étude et de Lutte contre les Discrimination (GELD),

Les sociologues et les minorités : connaissance et idéologie, Sociologie et Sociétés, volume 15, n² 2, p. 9-21.

Ethnie. Pluriel Recherches, Vocabulaire historique et critique des relations inter-ethniques, $\mathrm{n}^{\circ} 1$, p. 50-51.

Pour une sociologie des relations interethniques et des minorités, Rennes : PUR.

Migrations internationales et relations interethniques. Recherches, politique et société, Paris : L'Harmattan, p. 345-354.

The Ethnical Revival in the Modern World, Cambridge : Cambridge University Press.

Dossier « Relations interethniques », vol. 4.

Les recherches interethniques en France : le renouveau?, Migrants-formation, $\mathrm{n}^{\circ} 109$, p. 48-65.

Mixité sociale et politique de peuplement : genèse de l'ethnicisation des opérations de réhabilitation, Sociétés contemporaines, n 33-34, p. 59-86. 
TERSIGNI S., 2008

TISSOT S., 2005

VAN DEN BERGHE P., 1967

VEI-Enjeux, 2002

WIEVIORKA M., 1998

WEBER M., 1995 [1922]

WIRTH L., 1945

X, MALCOLM, (1993 [1965])
« Pour quelques gouttes de sang ». Conflits de normes et déontologie médicale face à la circoncision et à l'hyménorraphie, Revue des Sciences Sociales, nº 39, p. 104-113.

Une « discrimination informelle ». Usages du concept de mixité sociale dans la gestion des attributions de logement HLM, Actes de la Recherches en Sciences sociales, $n^{\circ}$ 159, septembre, p. 54-69.

Race and racism, New York : John Wiley and Sons Inc.

Dossier Enseigner en milieu ethnicisé face à la discrimination, vol. hors série $n^{\circ} 6$.

La production institutionnelle du racisme, Hommes et Migrations, vol. 1211, p. 5-15.

Les relations communautaires ethniques, In : Économie et société. L'organisation et les puissances de la société dans leur rapport avec l'économie, Paris : Pocket, p. 124-144.

The problems of minority groups, In : The Science of Man in the World Crisis, R. Linto (red.). Morningside Heights, N.Y., Columbia University Press, p. 347-72.

L'autobiographie de Malcolm X, Paris, Press Pocket. 